\title{
Epidemiologia do Acidente Vascular Encefálico no Brasil
}

\author{
Epidemiology of Stroke in Brazil \\ Epidemiología del Accidente Cerebrovascular en Brasil
}

Adriano Júnior Lucarelli Margarido ${ }^{*}$, Ana Flávia Salgado Rodrigues Gomes, Gabriel Lucas Souza Araújo1, Marcella Ciotti Pinheiro', Leonardo Brandão Barreto'.

\section{RESUMO}

Objetivo: Analisar dados epidemiológicos do Acidente Vascular Encefálico (AVE) no Brasil nos últimos 5 anos. Métodos: Estudo ecológico de abordagem analítica entre agosto de 2015 a agosto de 2020 a partir de dados do Departamento de Informática do Sistema Único de Saúde (SUS) e Sistemas de Informações Hospitalares do SUS. Foram buscados dados acerca da morbidade e taxas de internação, além da produção hospitalar de tratamento do AVE. Ademais, foi feita revisão bibliográfica a partir das bases de dados PubMed e Scientific Eletronic Library Online (SciELO), para melhor interpretação dos dados. Resultados: Nos últimos cinco anos, houve um total de 422.638 internações por AVE. Observou-se maior internação masculina, entretanto, maior mortalidade feminina. Ademais, mostrou-se que o aumento da incidência e mortalidade é proporcional ao aumento da idade. Apesar da diminuição do número de óbitos, observou-se aumento da incidência da doença. Conclusão: Conclui-se que a taxa de incidência, internação por AVE e valor de serviços de saúde cresceram ao longo dos anos, se fazendo necessários pontos de melhoria na assistência à saúde no que diz respeito à prevenção e promoção.

Palavras-chave: Epidemiologia, Acidente vascular encefálico, Neurologia.

\begin{abstract}
Objective: To analyze epidemiological data on Cerebrovascular Accident (CVA) in Brazil in the last 5 years. Methods: Ecological study with an analytical approach between August 2015 and August 2020 based on data from the Information Technology Department of the Unified Health System (SUS) and SUS Hospital Information Systems. Data on morbidity and hospitalization rates were sought, in addition to the hospital production of stroke treatment. In addition, a bibliographic review was carried out from the PubMed and Scientific Electronic Library Online (SciELO) databases, for a better interpretation of the data. Results: In the last five years, there were a total of 422,638 admissions for stroke. There was greater male hospitalization, however, higher female mortality. Furthermore, it was shown that the increase in incidence and mortality is proportional to the increase in age. Despite the decrease in the number of deaths, there was an increase in the incidence of the disease. Conclusion: It is concluded that the incidence rate, hospitalization for CVA and the value of health services have grown over the years, making necessary points of improvement in health care with regard to prevention and promotion.
\end{abstract}

Key words: Epidemiology, Stroke, Neurology.

\section{RESUMEN}

Objetivo: Analizar datos epidemiológicos sobre Accidente Cerebrovascular (ACV) en Brasil en los últimos 5 años. Métodos: Estudio ecológico con abordaje analítico entre agosto de 2015 y agosto de 2020 con base

\footnotetext{
${ }^{1}$ Escola de Medicina Dinâmica do Vale do Piranga (FADIP), Ponte Nova - MG.

*E-mail: adrianolucam@gmail.com
} 
en datos del Departamento de Tecnología de la Información del Sistema Único de Salud (SUS) y Sistemas de Información Hospitalaria del SUS. Se buscaron datos sobre las tasas de morbilidad y hospitalización, además de la producción hospitalaria del tratamiento del accidente cerebrovascular. Además, se realizó una revisión bibliográfica de las bases de datos PubMed y Scientific Electronic Library Online (SciELO), para una mejor interpretación de los datos. Resultados: En los últimos cinco años, hubo un total de 422,638 ingresos por accidente cerebrovascular. Hubo mayor hospitalización masculina, sin embargo, mayor mortalidad femenina. Además, se demostró que el aumento de la incidencia y la mortalidad es proporcional al aumento de la edad. A pesar de la disminución en el número de muertes, hubo un aumento en la incidencia de la enfermedad. Conclusión: Se concluye que la tasa de incidencia, la hospitalización por ACV y el valor de los servicios de salud han crecido a lo largo de los años, haciendo necesarios los puntos de mejora en la atención de la salud en materia de prevención y promoción.

Palabras clave: Epidemiología, Ictus, Neurología.

\section{INTRODUÇÃO}

O Acidente Vascular Encefálico (AVE) é caracterizado porum conjunto de eventos cerebrovasculares, que ocorrem devido a uma disfunção presente na irrigação sanguínea cerebral de forma aguda e rápida. Pode ser dividido em duas categorias de acordo com a sua etiologia: isquêmico ou hemorrágico. O AVE isquêmico, é caracterizado por uma oclusão permanente ou transitória de uma artéria importante para irrigação e nutrição cerebral, podendo ser intracraniana, extracraniana, podendo se dar por conta de fenômenos trombóticos ou ateroembólicos ou mesmo a partir de trombose venosa. Já no que se refere ao AVE hemorrágico, ele ocorre quando há uma ruptura de uma artéria cerebral muitas vezes já acometida por lesões patológicas, como no caso dos aneurismas e malformações arteriovenosas (LIMA MJMR, et al., 2016).

Clinicamente, é difícil identificar a diferença entre esses dois tipos, isquêmico e hemorrágico, sendo esta normalmente a diferenciação se dá através de exames de neuroimagem, sendo considerado padrão ouro a tomografia computadorizada. Já a localização do AVE se associará também a uma série de diferenças na resposta do paciente, devido a mudanças das áreas cerebrais acometidas e suas apresentações. Dentre os sinais e sintomas, podemos relatar alguns exemplos como perda da visão, ataxia, distúrbios de sensibilidade, vertigem, disartria, confusão e dificuldade motora (MORAES MM, 2021).

Em relação ao desenvolvimento do AVE, pessoas que possuem os fatores de risco têm maior predisposição a serem acometidas, e dentre esses fatores de risco podemos citar os seguintes: hipertensão, diabetes mellitus, cardiopatias, ataque isquêmico transitório prévio, obesidade, etilismo, tabagismo, hipercolesterolemia, hiperuricemia, infecções, fatores genéticos, uso de contraceptivos hormonais, enxaqueca e fatores pró-trombóticos. Ainda há uma observação que esses episódios ocorrem com maior incidência em indivíduos acima de 40 anos, justamente porserem observada maior prevalência de tais fatores de risco (CORREIA JP et al., 2018).

Em relação aos mecanismos, o diabetes e o tabagismo são precursores das lesões endoteliais, das quais se originam as placas de ateroma, que quando rompidas podem causar estenose no lúmen dos vasos, acarretando em isquemia e, consequentemente, aumentando consideravelmente o risco de o paciente desenvolver o acidente vascular encefálico. Já a hipertensão e obesidade estão relacionados ao aumento da pressão na parede dos vasos sanguíneos e formação de placas de ateroma, respectivamente. Ambos são fatores que podem gerar AVE isquêmico, entretanto, a hipertensão também pode estar relacionada com o hemorrágico, já que pode ocorrer rompimento dos vasos cerebrais, principalmente quando em conjunto com outros fatores de risco (CORREIA JP et al., 2018).

No mundo, cerca de 17 milhões de pessoas morrem ao ano devido a doenças cardiovasculares, nas quais está incluso o AVE, o qual será uma das maiores causas de morte e de necessidade de reabilitação na maior parte das populações, chegando a cerca de 15 milhões de pessoas acometidas ao ano. Ainda, cerca de três milhões de mulheres e 2,5 milhões de homens morrem ao ano, sendo a terceira causa mais comum de óbito 
em países desenvolvidos, ficando atrás somente de doenças coronarianas e câncer (MAMED SN et al., 2019). Em relação ao aumento do número de casos em mulheres nos últimos anos, possui relação com a dupla jornada de trabalho extenuante com alimentação precária e maus hábitos de saúde, ainda com a adição dos contraceptivos hormonais, que podem aumentar o risco de eventos tromboembólicos (CORREIA JP et al., 2018).

Outro fator de importante discussão, devido ao fato de ser um fator de risco com possibilidade de ação é a relação como uso de drogas ilícitas, principalmente por pessoas mais jovens. Essas substâncias aumentam o risco de desenvolvimento do AVE devido a vasoconstrição, e em grandes doses podem facilmente causar eventos cardiovasculares, sendo um possível fator desencadeador do AVE isquêmico (GERZSON LR, et al., 2018).

No Brasil o AVE chega a ser a segunda causa de morte no Brasil, ultrapassando doenças oncológicas; o Ministério da Saúde estima uma taxa de 100 mil mortes ao ano. No ano de 2016 foram registradas 188.223 internações para tratamento de AVE isquêmico e hemorrágico (MAMED SN et al., 2019).

A problemática ainda aumenta quando observamos que o AVE não af eta somente a saúde, mas também a economia: na Argentina e Brasil foram gastos cerca de 900 milhões de dólares com assistência hospitalar dos pacientes com AVE. Além disso, os fatores de risco também possuem papel importante nesse âmbito, já que são estimadas perdas de cerca de 200 bilhões de dólares por ano com custos de saúde para fumantes, sendo apenas um contribuinte (LOPES JM et al., 2016).

Feigin VL, et al. (2009) e Heuschmann PU, et al. (2009) observaram que na Europa existem diferenças regionais em relação à incidência do $\mathrm{AVE}$, relatando que o leste europeu apresenta taxas menores, principalmente devido a estratégias de promoção da saúde e prevenção dos principais fatores de risco envolvidos no seu desenvolvimento.

Já no Brasil, houve a implementação do HIPERDIA, o programa de assistência à hipertensão arterial sistêmica e diabetes mellitus, que tem seu funcionamento baseado no tratamento medicamentoso e não medicamentoso. Entretanto, há uma carência de estudos no que se refere aos resultados de longo prazo deste programa, visto que foi desenvolvido em 2002, e ainda suas repercussões em doenças cardiovasculares, principalmente o AVE (LOPES JM et al., 2016).

Dessa forma, torna-se necessário focar em estratégias para diminuição das taxas de AVE e principalmente de rastreamento e prevenção de seus riscos, a fim da promoção de saúde à população e redução da taxa de mortalidade e custos envolvidos com a doença, visto que, os números obtidos mediante ao estudo foram altos. Portanto, o objetivo deste estudo foi analisar dados epidemiológicos do AVE no Brasil a fim de direcionar ações para prevenção do mesmo, identificar número de pacientes internados e taxa de mortalidade nos últimos 5 anos.

\section{MÉTODOS}

Trata-se de um estudo ecológico baseado em desenho de séries temporais e abordagem analítica entre agosto de 2020 e agosto de 2015, compreendendo informações de todas as unidades federativas do Brasil, a partir de observação de dados de internação de AVE pelas Autorizações de Internações Hospitalares (AlH) pela doença.

É um estudo que utiliza dados secundários provenientes do Sistemas de Informações Hospitalares do SUS (SIH) a partir do Departamento de Informática do SUS (DATASUS), sem identificação pessoal e aberto à consulta pública, não sendo necessária a submissão do mesmo ao Comitê de Ética em Pesquisa.

Utilizou-se como base para o diagnóstico de AVE a décima versão da Classificação Internacional de Doenças (CID-10) a partir do código 164, indicado como Acidente Vascular Cerebral não especificado hemorrágico ou isquêmico. A partir disso, foi feita busca na aba Morbidade Hospitalar do SUS por local de residência a partir de 2008, a fim de estratificação correta dos dados hospitalares não somente quanto à 
região, mas também levando em consideração faixa etária, sexo e cor/raça. A pesquisa seguiu os seguintes critérios relatados no Quadro 1.

Quadro 1 - Descrição dos dados utilizados para pesquisa da morbidade hospitalar do SUS por local de residência nos casos de AVC não especificado hemorrágico ou isquêmico.

\begin{tabular}{|l|l|}
\hline Linha & Região \\
\hline Coluna & Não ativa \\
\hline Conteúdo & $\begin{array}{l}\text { Internações, valor serviços hospitalares, média permanência, óbitos, taxa } \\
\text { mortalidade }\end{array}$ \\
\hline Períodos disponíveis & Agosto de 2020 a Agosto de 2015 \\
\hline Caráter de Atendimento & Todas as categorias \\
\hline Capítulo CID-10 & IX- Doenças do Aparelho Circulatório \\
\hline Lista Morbidades CID-10 & Acidente Vascular Cerebral não especificado hemorrágico ou isquêmico \\
\hline Faixa Etária 1 & 40 a 79 anos \\
\hline Sexo & Masculino e Feminino \\
\hline Cor/Raça & Branca, parda e preta \\
\hline
\end{tabular}

Fonte: Margarido AJL, et al., 2021; dados extraídos em: Ministério da Saúde (2020).

Além disso, foram ainda enquadrados procedimentos dos dados de produção hospitalar correspondentes a "Tratamento de Acidente Vascular Cerebral (AVC) (Isquêmico ou Hemorrágico Agudos)" e "Tratamento de Acidente Vascular Cerebral Isquêmico Agudo com Uso de Trombolítico", visto que o tratamento também se torna um importante indicador de saúde. Os dados foram obtidos a partir dos Procedimentos Hospitalares do SUS por local de residência no Brasil, incluindo os seguintes critérios especificados no Quadro 2.

Quadro 2 - Descrição dos dados utilizados correspondentes aos procedimentos hospitalares do SUS por local de internação no Brasil nos casos de Tratamento do AVC Isquêmico ou Hemorrágico Agudo e Isquêmico com uso de trombolítico.

\begin{tabular}{|l|l|}
\hline Linha & Região \\
\hline Coluna & Não ativa \\
\hline Conteúdo & $\begin{array}{l}\text { Internações, valor serviços hospitalares, média permanência, óbitos, taxa } \\
\text { mortalidade }\end{array}$ \\
\hline Períodos disponíveis & Agosto de 2020 a Agosto de 2015 \\
\hline Caráter de Atendimento & Todas as categorias \\
\hline Procedimento & $\begin{array}{l}\text { 0303040149 - Tratamento de Acidente Vascular Cerebral (AVC) (Isquêmico ou } \\
\text { Hemorrágico Agudo) } \\
\text { 0303040300 - Tratamento de Acidente Vascular Cerebral Isquêmico Agudo com } \\
\text { Uso de Trombolítico }\end{array}$ \\
\hline
\end{tabular}

Fonte: Margarido AJL, et al., 2021; dados extraídos em: Ministério da Saúde (2020). 


\section{RESULTADOS E DISCUSSÃO}

De acordo com a Tabela 1, observa-se o número de internações por AVE hemorrágico ou isquêmico no período de 2015 a agosto de 2020, separado por regiões, nas faixas etárias de 40 a 79 anos, sexo masculino e feminino, cor branca, preta e parda. O valor hospitalar estimado por média do tempo de permanência, número de óbitos e a taxa de mortalidade também são analisados na Tabela 1.

Tabela 1- Morbidade Hospitalar do SUS por local de residência no Brasil, no caso de AVE não especificado hemorrágico ou isquêmico, nas faixas etárias de 40 a 79 anos, sexos masculino e feminino, cor branca, preta e parda no período entre agosto de 2015 a agosto de 2020.

\begin{tabular}{cccccc}
\hline Região & Internações & $\begin{array}{c}\text { Valor Serviço } \\
\text { Hospitalar }(\mathbf{R} \$)\end{array}$ & $\begin{array}{c}\text { Média } \\
\text { Permanência }\end{array}$ & Óbitos & $\begin{array}{c}\text { Taxa de } \\
\text { Mortalidade }\end{array}$ \\
\cline { 5 - 6 } Norte & 19.108 & $21.205 .618,13$ & 7,8 dias & 3.181 & 16,65 \\
Nordeste & 96.228 & $100.054 .932,70$ & 7,4 dias & 14.508 & 15,08 \\
Sudeste & 193.601 & $251.936 .361,38$ & 7,3 dias & 26.981 & 13,94 \\
Sul & 92.333 & $130.101 .809,34$ & 6,8 dias & 9.550 & 10,34 \\
Centro-Oeste & 21.368 & $31.557 .851,47$ & 7,1 dias & 3.211 & 15,03 \\
\hline Total & 422.638 & $534.586 .573,02$ & 7,2 dias & 57.431 & 13,59 \\
\hline
\end{tabular}

Fonte: Margarido AJL, et al., 2021; dados extraídos em: Ministério da Saúde (2020).

De acordo com a Tabela 2, descreve se a produção hospitalar do SUS para o tratamento do AVE isquêmico ou hemorrágico com o uso de trombolítico, no período de agosto de 2015 a agosto de 2020, separado por região de residência no Brasil, número de internações, valor hospitalar gasto em média pelo tempo de permanência do paciente. Além disso, o número de óbitos e a taxa de mortalidade também estão presentes.

Tabela 2 - Produção Hospitalar do SUS por local de residência no Brasil, para tratamento de AVE isquêmico ou hemorrágico agudo e tratamento do AVE isquêmico com uso de trombolítico, no período entre agosto de 2015 a agosto de 2020 .

\begin{tabular}{cccccc}
\hline Região & Internações & $\begin{array}{c}\text { Valor Serviço } \\
\text { Hospitalar }(\mathbf{R} \$\end{array}$ & $\begin{array}{c}\text { Média } \\
\text { Permanência }\end{array}$ & Óbitos & $\begin{array}{c}\text { Taxa de } \\
\text { Mortalidade }\end{array}$ \\
\cline { 5 - 6 } Norte & 56.487 & $59.979 .685,60$ & 7,9 dias & 10.181 & 18,02 \\
Nordeste & 272.128 & $301.009 .650,43$ & 7,9 dias & 46.975 & 17,26 \\
Sudeste & 407.948 & $525.396 .283,28$ & 8,1 dias & 68.145 & 16,70 \\
Sul & 195.182 & $257.527 .513,24$ & 7 dias & 24.853 & 12,73 \\
Centro-Oeste & 59.125 & $83.982 .900,83$ & 7,5 dias & 8.924 & 15,09 \\
\hline Total & 990.870 & $1.227 .896 .033,38$ & 7,8 dias & 159.078 & 16,05 \\
\hline
\end{tabular}

Fonte: Margarido AJL, et al., 2021; dados extraídos em: Ministério da Saúde (2020).

Há observação de uma diferença muito significativa de número de internações apenas por AVE e para realizar o tratamento do mesmo. Isso pode ocorrer principalmente devido a falha de preenchimento e 
passagem de informações dos serviços para os sistemas de informatização do SUS. Como esperado, os valores de todos os outros parâmetros também são maiores quando feita a comparação, apenas não possuindo muita alteração em relação à média de permanência.

A região Sudeste apresenta um número de internações para procedimentos cerca de duas vezes maior que a região Nordeste (segunda com maior número de internações). Isso pode ocorrer devido aos recursos disponíveis tanto no ambiente hospitalar, mas também na assistência pré-hospitalar para recorrer às vítimas do AVE, já que apenas $20,5 \%$ dos municípios possuem os Serviços de Atendimento Móvel de Urgência (MACHADO CV, et al., 2011).

Além disso, também é importante observar alguns dados específicos entre pessoas do sexo masculino e feminino e indivíduos de cor branca, preta e parda a partir da morbidade. Em relação à população feminina com faixa etária de 40 a 79 anos e cores branca, preta e parda existe uma média de permanência menor de 7,4 dias em relação a homens com os mesmos critérios, sendo estes 7,5 dias. Além disso, há um menor número de internações (262.874 para mulheres e 324.477 para homens) e consequentemente menores gastos com serviços hospitalares. Diante desses valores, como já esperado, também encontramos um menor número de óbitos (37.051 para mulheres e 44.420 para homens). Entretanto, a taxa de mortalidade enquanto nos homens é de 13,69 temos um valor de 14,09 para mulheres, o que deve ser um ponto de atenção, pois, apesar de menos acometida, o sexo feminino tem uma probabilidade maior de eventos negativos em decorrência do AVE.

Em relação a cor, há uma observação que indivíduos de cor branca possuem mais internações (193.500) e menor taxa de mortalidade $(12,05)$ em relação a indivíduos de corpreta (28.553 internações e 14,04 de taxa de mortalidade). Entretanto, ainda podemos observar uma diferença significativa em relação ao número total de óbitos, sendo menor em indivíduos de cor preta (4.010 vs. 23.313 em brancos). A média de permanência ainda apresenta uma diferença pouco significativa entre as duas cores.

Além disso, ainda podemos observar, como esperado, números maiores na cor parda, devido principalmente aos números de autodeclaração no Brasil. Existe um total de 200.585 internações, média de permanência de 7,5 dias, 30.108 óbitos e taxa de mortalidade de 15,01.

Um ponto muito importante a ser observado é a diferença de gastos em valor de serviços hospitalares entre essas três cores/raças. Existe uma diferença discrepante entre os indivíduos de cor preta, com gasto de $\mathrm{R} \$ 33.665 .155,88$. Ainda, apesar do maior número, os indivíduos de cor parda possuem um gasto de $\mathrm{R} \$ 241.969 .072,04$ e os de cor branca de $\mathrm{R} \$ 259.222 .345,10$. O que podemos constatar é uma desigualdade social grande em relação aos valores de serviço e consequentemente os recursos gastos para o p rocesso de internação e outros processos intra-hospitalares. Apesar de menor, a população de cor preta possui uma diferença muito significativa e desigual em comparação às duas outras cores, e a de cor branca, mesmo possuindo menos internações, possui mais recursos, como esperado, ressaltando as questões sociais e de cor presentes em nosso país (LAZZARINO Al, et al., 2013).

Como já provado, em relação às proporções de idade, há um acometimento progressivo e maior de internações e dos outros índices em indivíduos de 70 a 79 anos em comparação com os de 40 a 49 anos. 0 único parâmetro que não possui diferença é a média de permanência, que se mantém na faixa de 7,5 dias. Isso ocorre devido a progressão de doenças como fatores de risco, principalmente as crônicas, que exercem um grande papel no desenvolvimento do AVE (ABREU PG, et al., 2012).

Infelizmente, no Brasil os estudos de AVE a nível nacional são limitados, sendo observada maior prevalência de estudos locais, com menor alcance. Apesar disso, é importante observar que em território nacional foram implementados programas de prevenção de eventos cerebrovasculares e principalmente seus fatores de risco que desde 2000 vêm fazendo grande diferença nos números.

Existe uma observação que entre os anos de 2000 e 2009 houve uma tendência de queda da taxa de mortalidade por AVE, sendo padronizada para ambos os sexos. Ainda, houve redução contínua e linear entre indivíduos mais novos, entre 30 e 49 anos (não abordados neste estudo). 
Entretanto, um estudo de revisão mostra que, apesar das taxas de mortalidade serem decrescentes em decorrência de menores complicações (com base em programas de prevenção) e assistência hospitalar adequada, há uma tendência de aumento do número total de AVE em todo o mundo, devido às transições epidemiológicas e demográficas das populações (MAMED SN et al., 2019).

Além disso, outro estudo mostra que nas últimas décadas houve uma diminuição de $42 \%$ do número de AVE em países desenvolvidos, enquanto nos subdesenvolvidos houve um aumento de $100 \%$ das taxas (FEIGIN VL et al., 2009). É possível observar que isso se deve, principalmente, a prioridades governamentais e ausência de programas de prevenção e promoção de saúde que, apesar de existirem no Brasil, não existem muitos estudos em grande escala avaliando sua ef etividade e principalmente o entendimento e aplicação da população em relação aos mesmos.

Reis C e Faro A. (2019) relatam a importância de tais programas de prevenção com base nos fatores de risco de hipertensão arterial e intervenções sobre o tabagismo, a fim da melhoria dos cuid ados de saúde a nível primário. Entretanto, apesar do foco em doenças, sabemos que os fatores emocionais e ambientais também contribuem para o desenvolvimento de tais resultados, infelizmente não sendo foco de combate de tais programas (PICCINI RX, et al., 2012). O comportamento humano possui um ef eito muito grande sobre as comorbidades, não somente em relação ao entendimento dos programas voltados para as mesmas, mas principalmente em relação às realidades de vida (LAZZARINO AI, et al., 2013; RABETTI AC e FREITAS SFT, 2011).

Além de programas voltados para a prevenção geral, ainda é observado que a incidência do AVE aumenta progressivamente a cada década de vida, sendo importante o enfoque a cada faixa etária, visto que ainda existem limitações específicas presentes em cada uma em relação ao acesso à saúde (LAZZARINO AI, et al., 2013).

\section{CONCLUSÃO}

Apesar de uma queda nas taxas de mortalidade, o número de pacientes acometidos pelo AVE, internações e valor de serviços de saúde crescem ao longo dos últimos anos, principalmente quando diz respeito a faixas etárias acima de 40 anos. Apesar de existirem programas de promoção e prevenção de saúde no SUS, estudos de abrangência nacional devem ser realizados para mensurar os resultados dos mesmos e ainda buscar pontos de melhorias, principalmente às dores de cada faixa etária. O AVE é uma doença em ascensão devido ao crescimento dos fatores de risco cada vez mais presentes no dia a dia da população, e o declínio das taxas de mortalidade não significa que as outras taxas estão em mesmo patamar, esse declínio se deve apenas a assistência ao AVE que tem se tornado mais adequada com o avanço da medicina.

\section{REFERÊNCIAS}

1. CORREIA JP, et al. Investigação Etiológica do Acidente Vascular Cerebral no Adulto Jo vem. Medicina Interna, 2018; 25(3): 213-223.

2. FEIGIN VL, et al. Worldwide stroke incidence and early case fatality reported in 56 population-based studies: a systematic review. Lancet Neurol, 2009;8(4): 355-69.

3. GERZSON LR, et al. O impacto do acidente vascular cerebral na qualidade de vida de crianças e adolescentes. Fisioterapia e Pesquisa, 2018, 25(3): 241-250.

4. HEUSCHMANN PU, et al. Incidence of stroke in Europe at the beginning of the 21 st century. Stroke, 2009; 40(5): 1557-63.

5. LAZZARINO AI, et al. Low socioeconomic status and psychological distress as synergistic predictors of mo rtality from stroke and coronary heart disease. Psychosom Med., 2013;75(3): 311-6.

6. LIMA MJMR, et al. Fatores associados ao conhecimento dos adultos jovens sobre histórico familiar de Acidente Vascular Cerebral. Revista Latino-Am. Enfermagem, 2016, 24(2814).

7. LOPES JM, et al. Hospitalização por acidente vascular encefálico isquêmico no Brasil: estudo ecológico sobre possível im pacto da Hiperdia. Rev Bras Epidemiol, 2016;19(1):122-13.

8. MACHADO CV, et al. Serviço de Atendimento Móvel de Urgência : análise da política brasileira. Rev Saúde Pública, 2011;45(3):519-28. 
9. MAMED SN, et al. Perfil dos óbitos por acidente vascular cerebral não especificado após investigação de códigos garbage em 60 cidades do Brasil, 2017. Revista Brasileira de Epidemiologia, 2019;22(Suppl 3).

10. MINISTÉRIO DA SAÚDE. Banco de dados do Sistema Único de Saúde (DATASUS). Disponível em http://www.datasus.gov.br. Acessado em:26 de outubro de 2020.

11. MORAES MM, et al. Clinical characterization, disability, and mortality in people with strokes during 90 days. Revista Brasileira de Enfermagem, 2021,75(2).

12. PICCINI RX, et al. Promoção, prevenção e cuidado da hipertensão arterial no Brasil. Rev Saúde Pública, 2012; 46(3): 543-50.

13. RABETTI AC, FREITAS SFT. Avaliação das ações em hipertensão arterial sistêmica na atenção básica. Rev. Saúde Pública, 2011;45(2): 258-68.

14. REIS C, FARO A. Repercussões psicológicas após um acidente vascular cerebral (avc): uma revisão de liter atura. Psic., Saúde \& Doenças, 2019; 20(1):16-32.

15. ABREU PG, et al. Medidas morfológicas em função do peso e da idade da ave, por meio de imagens. Revista Brasileira de Engenharia Agrícola e Ambiental, 2012;16(7):795-801. 